International Mathematical Forum, Vol. 8, 2013, no. 31, 1503 - 1508

HIKARI Ltd, www.m-hikari.com

http://dx.doi.org/10.12988/imf.2013.37149

\title{
A Note on Sandwich Engel Conditions on Lie Ideals in Semiprime Rings
}

\author{
Francesco Rania
}

Department of Legal, Historical, Economic and Social Sciences

Magna Graecia University of Catanzaro

Campus loc. Germaneto, Viale Europa, 88100 Catanzaro, Italy

raniaf@unicz.it

Copyright (c) 2013 Francesco Rania. This is an open access article distributed under the Creative Commons Attribution License, which permits unrestricted use, distribution, and reproduction in any medium, provided the original work is properly cited.

\begin{abstract}
Let $R$ be a prime ring of characteristic $\neq 2$ with a derivation $d \neq 0$, $L$ a Lie ideal of $R, k, m, n$ positive integers such that $v^{m}[d(u), u]_{k} v^{n}=0$, for all $u, v \in L$. We prove that $L$ must be central. We also examine the case $R$ is a 2 -torsion free semiprime ring and $[z, t]^{m}[d([x, y]),[x, y]]_{k}[z, t]^{n}=$ 0 , for all $x, y, z, t \in R$.
\end{abstract}

\section{Introduction}

Let $R$ be a prime ring, $Z(R)$ its center, $U$ its left Utumi quotient ring, $C$ the center of $U$ (usually called the extended centroid of $R$ ) and $d$ a non-zero derivation of $R$. For basic definitions and properties of these objects we refer the reader to [1], [5] and [9].

A well known result of Posner [14] states that if the commutator $[d(x), x] \in$ $Z(R)$, the center of $R$, for any $x \in R$, then $R$ is commutative.

This theorem indicates how the global structure of a $\operatorname{ring} R$ is often tightly connected to the behaviour of additive mappings defined on $R$. Following this line of investigation, several authors have generalized the Posner's Theorem and studied the relationship between the structure of prime ring $R$ and the behavior of some additive mappings satisfying algebraic conditions on appropriate subsets of $R$. 
In [10] Lanski generalizes the result of Posner to a Lie ideal. To be more specific, the statement of Lanski's theorem is the following: let $R$ be a prime ring, $L$ a non-commutative Lie ideal of $R$ and $d \neq 0$ a derivation of $R$. If $[d(x), x]_{k}=0$, for all $x \in L$, then either $R$ is commutative, or $\operatorname{char}(R)=2$ and $R$ satisfies $s_{4}$, the standard identity in 4 variables.

Following this line of investigation, more recently in [15] we proved:

Theorem Let $R$ be a non-commutative ring of characteristic different from 2, with center $Z(R)$, Utumi quotient ring $U$ and extended centroid $C$. Let $G$ be a non-zero generalized derivation of $R, k \geq 1$ a fixed integer. If $\left[G\left(\left[r_{1}, r_{2}\right]_{k}\right),\left[r_{1}, r_{2}\right]_{k}\right]=0$ for all $r_{1}, r_{2} \in R$, then one of the following holds:

1. there exists $\alpha \in C$ such that $G(x)=\alpha x$, for all $x \in R$;

2. $R$ satisfies the standard identity $s_{4}\left(x_{1}, \ldots, x_{4}\right)$ and there exist $a \in U$, $\alpha \in C$ such that $G(x)=a x+x a+\alpha x$, for all $x \in R$.

Here we will examine what happens in case $v^{m}[d(u), u]_{k} v^{n}=0$, for any $u, v \in L$, a Lie ideal of $R$ and $k, m, n \geq 1$ fixed integers. In all that follws we always assume that $\operatorname{char}(R) \neq 2$. We will prove that

Theorem 1.1 Let $R$ be a prime ring of characteristic $\neq 2$ with a derivation $d \neq 0, L$ a non-central Lie ideal of $R, k, m, n$ positive integers such that $v^{m}[d(u), u]_{k} v^{n}=0$, for all $u, v \in L$. Then $L$ is central.

We then examine the case $R$ is a 2-torsion free semiprime ring. The result we obtain is:

Theorem 1.2 Let $R$ be a prime ring of characteristic $\neq 2$ with a derivation $d \neq 0, k, m, n$ positive integers such that $[z, t]^{m}[d([x, y]),[x, y]]_{k}[z, t]^{n}=0$, for all $x, y, z, t \in R$. Then $R$ contain a non-zero central ideal.

Moreover there exists a central idempotent e of $U$ such that, on the direct sum decomposition $U=e U \oplus(1-e) U$, the derivation d vanishes identically on $e U$ and the ring $(1-e) U$ is commutative.

\section{Main results}

In all that follows, unless stated otherwise, $R$ will be a prime ring of characteristic $\neq 2, L$ a Lie ideal of $R, d \neq 0$ a derivation of $R$ and $n \geq 1$ a fixed integer such that $[d(x), x]^{n} \in Z(R)$, for all $x \in L$.

For any $\operatorname{ring} S, Z(S)$ will denote its center, and $[a, b]=a b-b a,[a, b]_{k}=$ $\left[[a, b]_{k-1}, b\right], a, b \in S$.

We will also make frequent use of the following result due to Kharchenko [8] (see also [12]): 
Let $R$ be a prime ring, $d$ a non-zero derivation of $R$ and $I$ a non-zero two-sided ideal of $R$. Let $F\left(x_{1}, \ldots, x_{n}, d\left(x_{1}\right), \ldots, d\left(x_{n}\right)\right)$ a differential identity in $I$, that is

$$
F\left(r_{1}, \ldots, r_{n}, d\left(r_{1}\right), \ldots, d\left(r_{n}\right)\right)=0 \quad \forall r_{1}, \ldots, r_{n} \in I .
$$

One of the following holds:

1. either $d$ is an inner derivation in $U$, the left Utumi quotient ring of $R$, in the sense that there exists $q \in U$ such that $d=\operatorname{ad}(q)$ and $d(x)=$ $\operatorname{ad}(q)(x)=[q, x]$, for all $x \in R$, and $I$ satisfies the generalized polynomial identity

$$
F\left(r_{1}, \ldots, r_{n},\left[q, r_{1}\right], \ldots,\left[q, r_{n}\right]\right)=0
$$

2. or $I$ satisfies the generalized polynomial identity

$$
F\left(x_{1}, \ldots, x_{n}, y_{1}, \ldots, y_{n}\right)=0 \text {. }
$$

Proof of Theorem 1.1. Suppose $L$ is a non-central Lie ideal of $R$. Since we assume that $\operatorname{char}(R) \neq 2$, by a result of Herstein [6], $L \supseteq[I, R]$, for some $I \neq 0$, an ideal of $R$, and also $L$ is not commutative. Therefore we will assume throughout that $L \supseteq[I, R]$. Without loss of generality we can assume $L=[I, I]$.

Hence $[z, t]^{m}[d([x, y]),[x, y]]_{k}[z, t]^{n}=0$, for any $x, y \in I$, then $I$ satisfies the differential identity

$$
F(x, y, t, z, d(x), d(y))=[z, t]^{m}[[d(x), y]+[x, d(y)],[x, y]]_{k}[z, t]^{n} .
$$

If the derivation $d$ is not inner, by Kharchenko's theorem [8], I satisfies the polynomial identity

$$
F(x, y, t, z, u, v)=[z, t]^{m}[[u, y]+[x, v],[x, y]]_{k}[z, t]^{n}
$$

and in particular $R$ satisfies

$$
[z, t]^{m}[[u, y],[x, y]]_{k}[z, t]^{n} \text {. }
$$

Since the latter is a polynomial identity for $I$, and so for $R$ too, it is well known that there exists a field $K$ such that $R$ and $M_{l}(K)$, the ring of all $l \times l$ matrices over $K$, satisfy the same polynomial identities (see [7], page 57, page 89). Let $e_{i j}$ the matrix unit with 1 in $(i, j)$-entry and zero elsewhere. Suppose $l \geq 2$. If we choose in $(3)$

$$
z=e_{21}, \quad t=e_{12}, \quad u=e_{11}, \quad y=e_{12}, \quad x=e_{21}
$$

then we get the contradiction $0=(-1)^{m} 2^{k} e_{12}$. Therefore $l=1$ and so $R$ is commutative, a contradiction. 
Let now $d$ be an inner derivation induced by an element $q \in U$. Then, $I$ satisfies the generalized polynomial identity

$$
[z, t]^{m}[q,[x, y]]_{k+1}[z, t]^{n} .
$$

Since by [2] $I$ and $U$ satisfy the same generalized polynomial identities, we have that $U$ satisfies (4). Moreover, since $U$ remains prime by the primeness of $R$, replacing $R$ by $U$ we may assume that $q \in R$ and $C=Z(Q)$ is just the center of $R$. Note that $R$ is a centrally closed prime $C$-algebra in the present situation [4], i.e. $R C=R$. By Martindale's theorem in [13], $R C$ (and so $R$ ) is a primitive ring which is isomorphic to a dense ring of linear transformations of a vector space $V$ over $C$.

Assume first that $\operatorname{dim}_{C} V \geq 3$.

We want to show that, for any $v \in V, v$ and $q v$ are linearly $C$-dependent. Since if $q v=0$ then $\{v, q v\}$ is $C$-dependent, suppose that $q v \neq 0$. If $v$ and $q v$ are $C$-independent, since $\operatorname{dim}_{C} V \geq 3$, then there exists $w \in V$ such that $v, q v, w$ are also linearly independent. By the density of $R$, there exist $x, y \in R$ such that

$$
z v=0, \quad t v=w, \quad z w=v, \quad x v=v, \quad y v=v, \quad z q v=0, \quad t q v=w .
$$

These imply that

$$
[z, t] v=v, \quad[x, y] v=0, \quad[x, y] q v=q v, \quad[z, t] q v=v
$$

and, by (4),

$$
0=\left([z, t]^{m}[q,[x, y]]_{k+1}[z, t]^{n}\right) v=(-1)^{k} v \neq 0
$$

which is a contradiction.

So we can conclude that $v$ are $q v$ are linearly $C$-dependent, and standard argument shows that $q \in C$ and $d=0$, which contradicts our hypothesis.

Therefore $\operatorname{dim}_{C} V$ must be $\leq 2$. If $\operatorname{dim}_{C} V=1$ then $R$ is commutative and we have again a contradiction. Hence we assume $R$ is not commutative and $\operatorname{dim}_{C} V=2$, so that we may assume that $R \subseteq M_{2}(C)$, the ring of all $2 \times 2$ matrices over $C$, and moreover $M_{2}(C)$ satisfies the same generalized polynomial identity of $R$, in particular $M_{2}(C)$ satisfies (4).

Notice that for $[z, t]=\left[e_{12}, e_{21}\right]=e_{11}-e_{22}$, we have that both $[z, t]^{m}$ and $[z, t]^{n}$ is an invertivle matrix in $M_{2}(C)$. Thus, starting from (4) and for $[z, t]=$ $e_{11}-e_{22}$, it follows that $M_{2}(C)$ satisfies the generalized identity $[q,[x, y]]_{k+1}$. In this case, by [10], and since $R$ is not commutative, we get the contradiction that $\operatorname{char}(R)=2$. 
We conclude by studying the semiprime case. In all that follows $R$ will be a 2-torsion free semiprime ring.

In order to prove the main result of this section we will make use of the following facts:

Fact 1 ([1], proposition 2.5.1) Any derivation of a semiprime ring $R$ can be uniquely extended to a derivation of its left Utumi quotient ring $U$, and so any derivation of $R$ can be defined on the whole $U$.

Fact 2 ([3], page 38) If $R$ is semiprime then so is its left Utumi quotient ring. The extended centroid $C$ of a semiprime ring coincides with the center of its left Utumi quotient ring.

Fact 3 ([3], page 42) Let $B$ be the set of all the idempotents in $C$, the extended centroid of $R$. Assume $R$ is a B-algebra orthogonal complete. For any maximal ideal $P$ of $B, P R$ forms a minimal prime ideal of $R$, which is invariant under any derivation of $R$.

Proof of Theorem 1.2. Since $R$ is semiprime, by Fact 2, $Z(U)=C$, the extended centroid of $R$, and, by Fact 1, the derivation $d$ can be uniquely extended on $U$. Since $U$ and $R$ satisfy the same differential identities (see [12]), then

$$
[z, t]^{m}[d([x, y]),[x, y]]_{k}[z, t]^{n}=0, \quad \forall x, y, z, t \in R .
$$

Let $B$ be the complete boolean algebra of idempotents in $C$ and $M$ be any maximal ideal of $B$.

Since $U$ is a B-algebra orthogonal complete (see (2) of Fact 1 in [3]), by Fact $3, M U$ is a prime ideal of $U$, which is d-invariant. Denote $\bar{U}=U / M U$ and $\bar{d}$ the derivation induced by $d$ on $\bar{U}$. For any $\bar{x}, \bar{y}, \bar{z}, \bar{t} \in \bar{U}$, and bu relation (5),

$$
[\bar{z}, \bar{t}]^{m}[d([\bar{x}, \bar{y}]),[\bar{x}, \bar{y}]]_{k}[\bar{z}, \bar{t}]^{n}=\overline{0} .
$$

In particular $\bar{U}$ is a prime ring and so, by Theorem 1.1 , either $\bar{d}=0$ in $\bar{U}$ or $[\bar{U}, \bar{U}]$ is central in $\bar{U}$, that is $\bar{U}$ is commutative. This implies that, for any maximal ideal $M$ of $B, d(U) \subseteq M U$ or $[U, U] \subseteq M U$. In any case both $[d(U), U] \subseteq M U$ and $d(U)[U, U] \subseteq M U$, for all $M$. Therefore $[d(U), U] \subseteq$ $\bigcap_{M} M U=0$ and $d(U)[U, U] \subseteq \bigcap_{M} M U=0$.

By using the theory of orthogonal completion for semiprime rings (see [1], chapter 3]), it follows that there exists a central idempotent element $e$ in $U$ such that on the direct sum decomposition $e U \oplus(1-e) U, d$ vanishes identically on $e U$ and the ring $(1-e) U$ is commutative.

Moreover, since $[d(U), U]=0$, we also have $[d(R), R]=0$. Therefore, by [11], it follows that $R$ contains a non-zero central ideal. 


\section{References}

[1] K.I. Beidar, W.S. Martindale, V. Mikhalev, Rings with generalized identities, Pure and Applied Math., Dekker, New York, 1996.

[2] C.L. Chuang, GPI's having coefficients in Utumi quotient rings, Proc. Amer. Math. Soc., 103(3) (1988), 723-728.

[3] C.L. Chuang, Hypercentral derivations, J. Algebra, 166 (1994), 34-71.

[4] J.S. Erickson, W.S. Martindale III, J.M. Osborn, Prime nonassociative algebras, Pacific J. Math., 60 (1975), 49-63.

[5] C. Faith, Lecture on Injective Modules and Quotient Rings, Lecture Notes in Mathematics, 49, Springer Verlag, New York, 1967.

[6] I.N. Herstein, Topics in ring theory, Univ. Chicago Press, 1969.

[7] N. Jacobson, PI-algebras, an introduction, Lecture notes in Math., 441, Springer Verlag, New York, 1975.

[8] V.K. Kharchenko, Differential identities of prime rings, Algebra and Logic, 17 (1978), 155-168.

[9] J. Lambek, Lecture on Rings and Modules, Blaisdell Waltham, MA, 1966.

[10] C. Lanski, An Engel condition with derivation, Proc. Amer. Math. Soc., 118(3) (1993), 731-734.

[11] C. Lanski, An Engel condition with derivation for left ideals, Proc. Amer. Math. Soc., 125(2) (1997), 339-345.

[12] T.K. Lee, Semiprime rings with differential identities, Bull. Inst. Math. Acad. Sinica, 20(1) (1992), 27-38.

[13] W.S. Martindale III, Prime rings satisfying a generalized polynomial identity, J. Algebra, 12 (1969), 576-584.

[14] E.C. Posner, Derivations in prime rings, Proc. Amer. Math. Soc., 8 (1957), 1093-1100.

[15] F. Rania, An Engel Condition with Generalized Derivations on $k$-th Commutators, Int. Math. Forum, 4 (2009), 113-118.

Received: July 24, 2013 